\title{
Establishment of lateral organ asymmetries in the invertebrate chordate, Ciona intestinalis
}

\author{
Karl Palmquist and Brad Davidson * (1)
}

\begin{abstract}
Background: The evolutionary emergence and diversification of the chordates appear to involve dramatic changes in organ morphogenesis along the left/right axis. However, the ancestral chordate mechanism for establishing lateral asymmetry remains ambiguous. Additionally, links between the initial establishment of lateral asymmetry and subsequent asymmetries in organ morphogenesis are poorly characterized.

Results: To explore asymmetric organ morphogenesis during chordate evolution, we have begun to characterize left/right patterning of the heart and endodermal organs in an invertebrate chordate, Ciona intestinalis. Here, we show that Ciona has a laterally asymmetric, right-sided heart. Our data indicate that cardiac lateral asymmetry requires $\mathrm{H}^{+}$/ $\mathrm{K}^{+}$ion flux, but is independent of Nodal signaling. Our pharmacological inhibitor studies show that ion flux is required for polarization of epidermal cilia and neurula rotation and suggest that ion flux functions synergistically with chorion contact to drive cardiac laterality. Live imaging analysis revealed that larval heart progenitor cells undergo a lateral shift without displaying any migratory behaviors. Furthermore, we find that this passive shift corresponds with the emergence of lateral asymmetry in the endoderm, which is also ion flux dependent.

Conclusions: Our data suggest that ion flux promotes laterally asymmetric morphogenesis of the larval endoderm rudiment leading to a passive, Nodal-independent shift in the position of associated heart progenitor cells. These findings help to refine hypotheses regarding ancestral chordate left/right patterning mechanisms and how they have diverged within invertebrate and vertebrate chordate lineages.
\end{abstract}

Keywords: Left/right patterning, Chordate evolution, Tunicates, Heart development, Endoderm development, Nodal signaling

\section{Background}

The emergence of diverse bilaterian body plans is thought to reflect variable deployment of developmental regulatory modules along ancestral signaling axes. Widely divergent bilaterian clades appear to deploy the same set of signaling pathways to coordinate embryonic growth along a cartesian axial system. The anterior/posterior axis is dictated by Wnt signaling, dorsal/ventral by BMP signaling, and left/right (LR) by Nodal signaling. Broad conservation of axial signaling is thought to reflect a deeply

${ }^{*}$ Correspondence: bdavids1@swarthmore.edu

Department of Biology, Swarthmore College, 500 College Ave., Swarthmore, PA 19081, USA homologous, ancestral embryonic patterning mechanism at the base of the bilateria. However, the process by which the initial axial signals coordinate subsequent organ morphogenesis and how this process has been altered to generate novel traits remains poorly defined. This is particularly true for the more recently characterized LR molecular axis [1]. Insights into LR axis specification are essential for deciphering patterns of evolutionary diversification. Additionally, errors in embryonic LR axis formation underlie prevalent congenital abnormalities in organ form and function $[2,3]$.

Left-right patterning is a complex, multi-step process [4]. First, lateral symmetry must be broken. This initial asymmetry then generates a stable lateralized signaling 
gradient that is subsequently relayed to adjacent tissues. Finally, lateral differences in exposure to axial signals drive differential programs of organ morphogenesis. Evolutionary conservation of this complex pathway is stage specific with an hourglass-like conformation, as stringent conservation appears to occur primarily in the central Nodal signaling step. At the bottom of the hourglass, there is enormous variability of morphogenetic programs directed by axial signals. At the top of the hourglass, variability in the initial symmetry-breaking mechanisms, even within the vertebrates, has derailed attempts to reconstruct ancestral LR patterning mechanisms. In Xenopus, zebrafish and mouse, symmetry is broken by ciliary flow [5-7]. Polarized cilia drive asymmetric flow across a midline LR organizer tissue, termed the gastrocoel roof plate in Xenopus [7], the node in mice [5, 8] or Kupffer's vesicle in Zebrafish [6]. Flow across the LR organizer generates localized signaling gradients including fibroblast growth factor (FGF), Sonic hedgehog (SHH), Nodal and $\mathrm{Ca}^{2+}$ ions [9-13]. Through a poorly characterized relay mechanism, this midline axial signal drives asymmetric Nodal signaling in the adjacent lateral plate mesoderm. In contrast, the initial symmetry disruption in chick and pig embryos appears to involve directed rotation of organizer tissue instead of ciliary flow [14]. Intriguingly, establishment of the initial LR asymmetry in both frog and chick embryos requires ion flux driven by a $\mathrm{H}^{+} / \mathrm{K}^{+}$-ATPase $[14,15]$. The role of ion flux in lateral patterning remains controversial. The initial work in Xenopus embryos indicated that asymmetric localization of the ATPase during early cleavage stages led to an early break in symmetry [15]. Recent studies contradict these results, suggesting that ion flux contributes to LR patterning by participating in node ciliogenesis [16]. $\mathrm{H}^{+} / \mathrm{K}^{+}$-ATPase dependent ion flux is also required for establishing lateral asymmetry in tunicates and sea urchins $[17,18]$. Studies in sea urchins and tunicates suggest that ion flux functions upstream of Nodal signaling during lateral patterning $[17,18]$. However, the specific role of ion flux in tunicate lateral organ morphogenesis has not been investigated.

Although the initial symmetry-breaking mechanisms vary, Nodal signaling is thought to play a highly conserved role in bilaterian left/right patterning preserved through 500 million years of evolutionary diversification. The initial studies of vertebrate LR patterning revealed a key role for Nodal and downstream expression of the transcription factor Pitx [12, 19-22]. Subsequent studies revealed that Nodal/Pitx also regulates LR patterning in the invertebrate chordates, including both amphioxus and tunicates $[23,24]$ and more broadly within non-chordate deuterostomes (sea urchins) [18] and protostomes (molluscs) [25]. Most recently, a Nodal-related gene was shown to regulate pitx expression and lateral branching in hydra [26], suggesting that Nodal-dependent lateral patterning represents an ancestral eumetazoan character state. In contrast, genes encoding Nodal are not represented in the ecdysozoan taxa [1]. There is also some evidence for alternative Nodal-independent mechanisms underlying LR patterning [27]. Despite these inconsistencies, Nodal's status as the linchpin of LR patterning has remained largely unchallenged and research has predominantly focused on deciphering upstream and downstream mechanisms.

Despite an intensive and productive research effort, numerous aspects of vertebrate left/right patterning remain poorly described. Crucial gaps include (1) the precise contributions of ciliary flow, mechanosensory cilium, $\mathrm{Ca}^{2+}$ ion flux and midline FGF, SHH and Nodal morphogen gradients in establishing an initial, robust signaling axis, (2) the relay system by which the initial midline gradient propagates to lateral portions of the embryo, (3) the cascading series of downstream effects that lateralize organ morphogenesis producing striking asymmetries in heart and gut formation. One highly productive line of inquiry regarding lateralized organogenesis focuses on zebrafish heart formation. As in all vertebrates, the zebrafish heart primordium begins as a midline structure. As the heart tube begins to form, it tilts to the left. Subsequently, the zebrafish heart undergoes lateralized looping, similar to that in other vertebrate embryos [28]. A series of studies has revealed the precise means by which Nodal signaling in the left lateral plate participates in a regulatory circuit with BMP and Nodal to drive tilting of the heart primordium [29, 30]. Intriguingly, subsequent looping morphogenesis has been revealed to be Nodal independent [27]. This raises the question of what alternative, parallel pathways may function in conjunction with Nodal to drive the full suite of lateralized morphogenetic cell behaviors. To further our understanding of vertebrate organ morphogenesis and the role of LR patterning in chordate evolution, we have initiated a study of LR heart patterning in an invertebrate chordate phylum, the tunicates.

Tunicates are the closest sister group to the vertebrates, making them a key taxon for studying chordate development and evolution. The best studied model of tunicate development is Ciona intestinalis. Ciona development shares fundamental similarities to that of vertebrate embryos, but within a much simpler cellular and genetic context, enabling clarification of complex shared processes [31]. In particular, the gene regulatory networks and morphogenetic cell behaviors underlying formation of the notochord, neural tube and cardiopharyngeal mesoderm share extensive similarities with orthologous processes in vertebrate embryos [32-37]. Recent studies have begun to produce a high-resolution map of the 
Ciona cardiopharyngeal gene regulatory network and delineate how this network regulates lineage specification and associated cell behaviors underlying early steps of heart development [38-42]. However, the differentiation of Ciona cardiomyocytes and subsequent morphogenesis of a functioning organ occurs after metamorphosis and remains poorly characterized. Additionally, early lineage specification and axis formation in Ciona have diverged from other chordates, relying heavily on maternal determinants associated with rapid embryogenesis. One consequence of this more "mosaic" developmental mode is a reduced role for key embryonic signaling pathways such as Nodal and BMP in early patterning. Thus, while Nodal signaling has a key organizer function in both vertebrate and amphioxus embryos, contributing to all three embryonic axes, Ciona Nodal only participates in early LR patterning [43].

Recent research has begun to elucidate the molecular mechanisms underlying left/right patterning in tunicates. The initial symmetry breakage appears to involve lateral rotation of the neurula stage embryos driven by ectodermal cilia and subsequent lateralized contact with the surrounding chorion $[44,45]$. Dechorionation disrupts this process, revealing that chorion contact is required for laterally asymmetric expression of nodal in the ectoderm and downstream expression of pitx. Disruption of Nodal signaling has been shown to randomize markers of tunicate embryonic lateral asymmetry including directional tail bending and lateralized positioning of a light sensing organ, the ocellus [44]. Disruption of $\mathrm{H}^{+} / \mathrm{K}^{+}$-ATPase function also leads to loss of asymmetric pitx expression in Ciona [17]. The impact of these manipulations on post-larval organ asymmetries, including the heart and gut, has not been investigated. Indeed, although tunicate organ asymmetries have been repeatedly noted in the literature, whether these asymmetries represent robust directed lateralities has not been investigated.

To unlock the potential use of Ciona to investigate laterally asymmetric organ morphogenesis during chordate evolution, we have begun to characterize left/right patterning of the heart and endodermal organs. Here, we show that Ciona has a laterally asymmetric, rightsided heart. Our data indicate that this lateral asymmetry requires $\mathrm{H}^{+} / \mathrm{K}^{+}$ion flux and is independent of Nodal signaling. We propose that ion flux-dependent cilia polarization leads to laterally asymmetric endoderm morphogenesis that drives a passive shift in heart progenitor position.

\section{Methods}

\section{Embryological techniques}

Ciona (Ciona robusta/Ciona intestinalis type a) [46] adults were collected by M-Rep (San Diego County) and kept at $18{ }^{\circ} \mathrm{C}$ in artificial seawater (Crystal Sea Marine Mix) under constant light. Fertilization, dechorionation and electroporation were performed as previously described [47]. All electroporations were performed using $100 \mu \mathrm{g}$ of each DNA construct to ensure high penetrance. Rearing of juveniles was performed by settling hatching larva (St. 29) on non-coated dishes and allowing development until 4 days post-fertilization (d.p.f.) at $18^{\circ} \mathrm{C}$.

\section{Inhibitor treatments}

Omeprazole (PHR1059, Sigma, $10 \mathrm{mM}$ stock) and SB431542 (S4317, Sigma, $30 \mathrm{mM}$ stock) inhibitor stock dilutions were made using DMSO (D2650, Sigma). All the final dilutions were made by introducing the appropriate volume of stock inhibitor to $10 \mathrm{~mL}$ of filtered seawater (FSW) containing embryos. For chorionated embryos, a "spin-down" approach was used to wash off omeprazole. Treated FSW and embryos were placed in $10-\mathrm{mL}$ centrifuge tubes and spun on hand centrifuge 40 times, rinsed, centrifuged 40 times again and rinsed once more in FSW. Dechorionated embryos were rinsed using a transfer pipette three times into fresh dishes with $10 \mathrm{~mL}$ FSW.

\section{Staining and confocal microscopy}

Staining for larva and juveniles was performed using an overnight fixation in $0.4 \%$ paraformaldehyde in FSW (16\% stock; Electron Microscopy Sciences). For phalloidin staining, larva and juveniles were incubated for 5 min in $0.1 \%$ Triton X-100 (Sigma) in FSW. Samples were then washed in $1 \times$ PBS $+1 \%$ BSA (PBS-B) and $1 \times$ PBS $+0.1 \%$ Triton (PBTr), followed by two washes in PBS-B. Samples were then incubated on a nutator overnight at $4{ }^{\circ} \mathrm{C}$ in $250 \mu \mathrm{L}$ PBS-B $+1: 125$ Alexa Fluor 635 phalloidin (Invitrogen). This incubation was followed by two rinses in $1 \times$ PBS-B, and samples were mounted in glycerol using a raised coverslip.

Staining for cilia was performed using overnight methanol fixation at $-20{ }^{\circ} \mathrm{C}$. Embryos were then rinsed in $\mathrm{MeOH} / 1 \times \mathrm{PBS}+0.1 \%$ Tween-20 (PBTw). Samples were then rinsed $5 \times$ in $\mathrm{PBTw}$, followed by a $1-\mathrm{h}$ incubation in PBS-B at room temperature. Samples are then incubated overnight on a nutator at $4{ }^{\circ} \mathrm{C}$ in PBS-B $+1: 1000$ anti-gamma-tubulin (T5326, Sigma) and 1:1000 antiacetylated tubulin (T7451, Sigma). Samples are then washed $3 \times$ in PBTw and then blocked using $2 \%$ normal donkey serum $(5 \% \mathrm{v} / \mathrm{v}$; Jackson ImmunoResearch 017-000-121) for $1 \mathrm{~h}$. Samples are then nutated in $1 \times$ PBS $+2 \%$ NDS $+1: 1000$ donkey anti-mouse 488 (Invitrogen) and 1:1000 donkey anti-mouse 633 (Invitrogen) overnight at $4{ }^{\circ} \mathrm{C}$. Embryos were then washed $3 \times$ in $1 \times$ PBS-B and mounted in glycerol. Z-stack images $(1-\mu \mathrm{m}$ 
sections for juveniles and $0.5-\mu \mathrm{m}$ sections for larva and embryos) were acquired using a Leica SP5 confocal microscope.

\section{Larval heart positioning assay}

Fixed larva were mounted in $75 \%$ glycerol in between a large and small coverslip and then rotated by moving the top coverslip so the ventral surface was facing the inverted microscope objective. Anterior was demarcated by pigment spots and the midline by the larval endostyle rudiment. In all larvae sampled, the middle of the endostyle rudiment was positioned equidistant from both the left and right sides of the larval head, and the line bisecting the larvae from the middle of the endostyle to the middle of the tail demarcated the midline. Using ImageJ $(\mathrm{NIH})$, the distance in micrometer from the middle of the heart cells to the nearest side of the head was measured. We also measured the distance from the middle of the larval head to side of the head containing the heart progenitors. The latter distance was divided by the former distance to provide a cell shift ratio (Fig. 2), and heart cell positioning was categorized based on this ratio.

\section{Quantification of cilia positioning and length}

For quantification of cilia length, images were processed in ImageJ. Cilia were measured from the base of the cilium to the tip, to the nearest hundredth of a micrometer.

For quantification of cilia polarization, cells were measured from the anterior-most point to the posterior-most point. Then, the distance from a cilium's base to the posterior-most point was measured. If the distance from a cilium's base to the posterior-most point was either equal to or less than a third the distance from the cell's anterior-most point to posterior-most point, these cilia were classified as posteriorly polarized.

\section{Statistical analysis}

All analyses were performed in Excel. All $n$ values are reported in the text. $P$ values are reported in the figure legends for $\chi^{2}$ analysis, Wilcoxon rank sum test and student's $t$ test (two sample, unpaired, unequal variance).

\section{Results}

\section{Directed lateral asymmetry of the Ciona heart}

The recent characterization of Ciona embryonic leftright patterning has failed to ascertain if embryonic asymmetry translates into post-metamorphic asymmetry. Therefore, we examined heart position in Ciona juveniles. We assessed juvenile heart laterality using four morphological markers: the endostyle, pigments spots, oral siphon and stalk-like ampulla (Fig. 1a). The endostyle and pigment spots demarcate the ventral and dorsal midlines, respectively, while the oral siphon and ampulla demarcate the anterior-posterior axis. Using these landmarks, we identified the heart as consistently right-sided in wild-type juveniles (Fig. 1a", e).

We next asked when heart field asymmetry first arises. In tailbud stage embryos, the heart precursors consist of bilateral clusters of heart progenitors that initially fuse symmetrically at the ventral midline [41]. However, heart progenitor positioning along the left-right axis during late tailbud and early larval stages has not been characterized. To fill this gap, we transgenically labeled heart progenitor cell nuclei using the Mesp > H2B:GFP reporter construct (the Ciona Mesp enhancer driving expression of a Histone 2B-GFP fusion protein) [33] and examined heart cell position in three stages: late tailbud II (St. 24, 13.5 HPF), late tailbud III (St. 25, 15.9 HPF) and late swimming larva (St. 29, $24 \mathrm{HPF}$ ) [48]. At St. 24, the head trunk region is roughly symmetric and the heart progenitor cells are symmetrically distributed along either side of the midline endostyle (Fig. 1c'). By St. 25, the trunk region displays laterally asymmetric lobes (Fig. $\left.1 \mathrm{c}^{\prime \prime}\right)$. This overall morphological asymmetry is associated with a lateral shift of the heart progenitor cells to one side of the endostyle (Fig. 1 $\mathrm{c}^{\prime \prime}$ ). During St. 29, the head elongates and the papillae mature, priming larvae for settlement. The heart progenitor pool has divided to produce a medial group of heart precursor cells and is flanked by two lateral clusters of atrial siphon muscle (ASM) precursors (Fig. $\left.1 \mathrm{c}^{\prime \prime \prime}\right)$. In these late larvae, cardiac laterality is more evident as the heart precursors have shifted completely off the endostyle-demarcated midline (Fig. 1c'"').

\section{Dechorionation disrupts lateral heart directionality}

We next asked whether chorion contact contributes to heart lateral asymmetry. Studies in the tunicate Halocynthia roretzi have shown that removal of the chorion disrupts embryonic left/right patterning leading to midline positioning of the tail and neural complex [44]. Further, studies in Halocynthia revealed that lateralized contact with the chorion is required for lateralized expression of nodal and subsequent Nodal-dependent embryonic LR patterning (Fig. 1d). In Ciona, dechorionation disrupts asymmetric gene expression, leading to bilateral nodal expression [17]. However, the impact of dechorionation on embryonic asymmetric morphogenesis has not been characterized. To determine if a chorion-dependent mechanism regulates embryonic LR asymmetry, we examined tail bending in chorionated and dechorionated embryos (St. 23) [48]. In chorionated controls, the tip of the tail was located to the right of the head in all embryos scored (50/50; two trials). When the chorion was removed, nearly half the embryos scored had a reversal in the direction of tail bending (29/60 left; 31/60 right; two trials). Thus, in accordance with data in Halocynthia [44], 

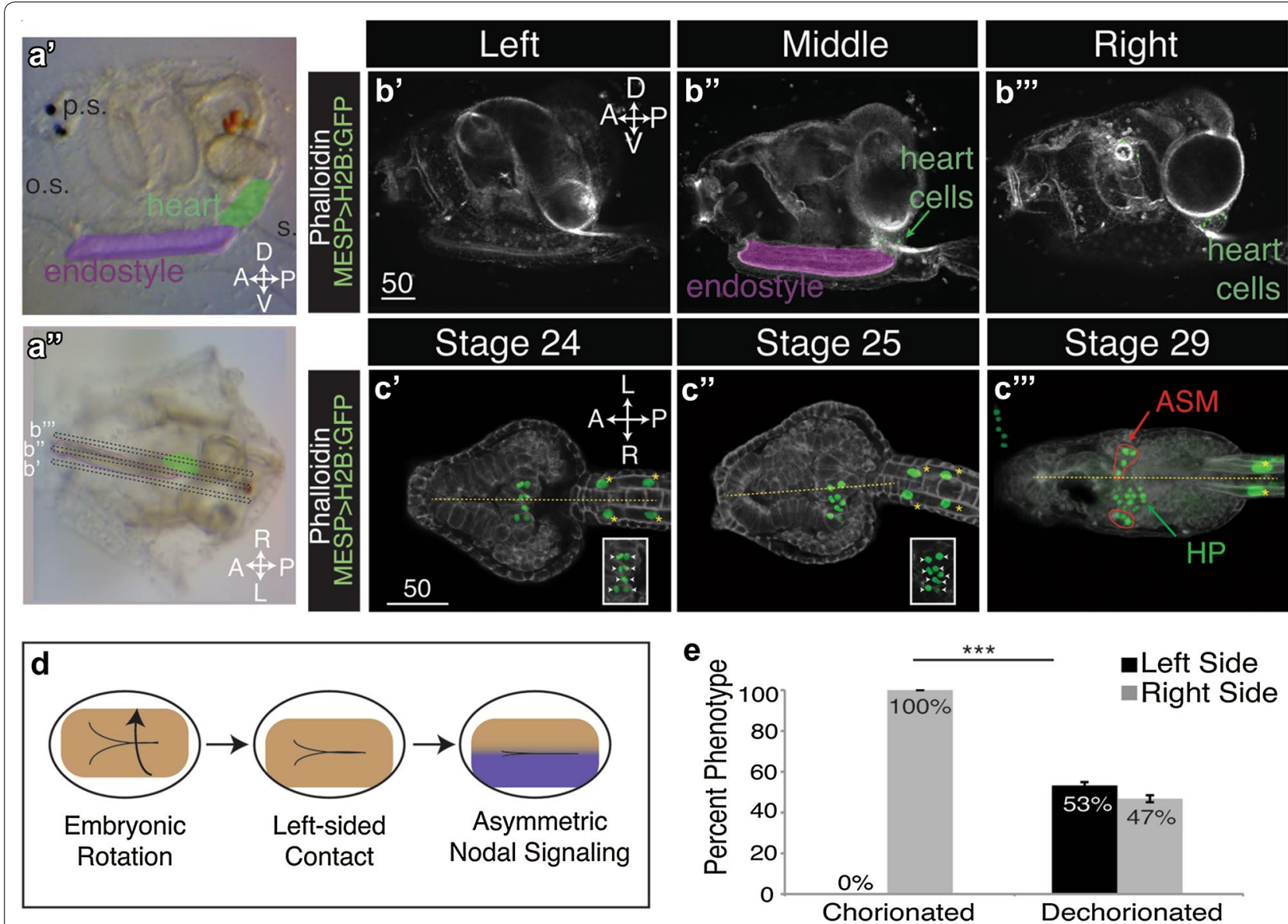

Fig. 1 Right-sided heart positioning in Ciona. $\mathbf{a}^{\prime}-\mathbf{a}^{\prime \prime}$ Lateral ( $\mathbf{a}^{\prime}$ ) and dorsal (a') views of a typical Ciona juvenile, (o.s.) oral siphon, (p.s.) pigment spots, (s.) stalk. Heart and endostyle are false-colored, ( $\left.\mathbf{b}^{\prime}-\mathbf{c}^{\prime \prime \prime}\right)$ lateral confocal sections of Ciona juveniles $\left(\mathbf{b}^{\prime}-\mathbf{b}^{\prime \prime \prime}\right)$ and ventral projections of late embryonic and larval stages $\left(\mathbf{c}^{\prime}-\mathbf{c}^{\prime \prime \prime}\right)$. Cell outlines are visualized using phalloidin, and heart founder cell nuclei are transgenically labeled by Mesp > H2B:GFP (green). Sections in ( $\left.\mathbf{b}^{\prime}-\mathbf{b}^{\prime \prime \prime}\right)$ correspond to lines in $\left(\mathbf{a}^{\prime \prime}\right)$. Lateral differences in GFP intensity (most evident in $\mathbf{c}^{\prime \prime}$ ) reflect uneven incorporation of transgenic constructs on the right versus left sides of the embryo. In $\left(\mathbf{c}^{\prime}-\mathbf{c}^{\prime \prime}\right)$, heart progenitor nuclei are shown at high gain in the bottom right and demarcated by white arrowheads. Atrial siphon muscle precursors (ASM) are circled in red, and anterior tail muscle (ATM) cells are marked using yellow asterisks. d Schematic of neurula rotation and hypothesized mechanism for establishing embryonic left-right axis, and dorsal views are shown. e Impact of dechorionation on juvenile heart positioning, and data represent three trials including 71 chorionated samples and 71 dechorionated samples. $P$ value $(1.4 \mathrm{E}-12)$ between chorionated and dechorionated samples was derived using a $x^{2}$ test. In all images and schematics, anterior is to the left. All scale bars are in micrometers

a chorion-dependent mechanism regulates Ciona lateral tail directionality (i.e., left- vs. right-sided bending). However, dechorionated Halocynthia embryos developed with straight tails [44] while dechorionated Ciona embryos never exhibited this laterally symmetric phenotype. The lack of midline tails in dechorionated Ciona embryos suggests that tail growth asymmetry is chorion independent. Alternatively, a physical barrier may block midline tail growth.

To determine whether Ciona heart laterality is influenced by a chorion-dependent mechanism, dechorionated zygotes were reared through metamorphosis and scored for heart positioning in 4-day-old juveniles.
Strikingly, nearly half the juveniles that developed from dechorionated eggs had a left-sided heart (Fig. 1e). These results suggest that the chorion is necessary for lateral heart directionality. However, no midline hearts were observed, indicating that a chorion-dependent mechanism does not contribute to asymmetric positioning. Alternatively, a physical barrier, such as the endostyle, may obstruct midline heart formation.

\section{Disruption of Nodal signaling does not perturb asymmetric heart positioning}

In both Halocynthia and Ciona, removal of the chorion disrupts the embryonic left/right Nodal signaling axis 
$[17,44]$. We therefore hypothesized that Nodal signaling regulates lateral heart asymmetry downstream of chorion contact. To test this hypothesis, we disrupted Nodal signaling by treating neurula stage embryos (St. 14) [48] with the well-established pharmacological inhibitor SB431542 [24, 27, 49] and examined the impact on both embryonic morphology (tail bending) and heart asymmetry. DMSO controls displayed normal right-sided tail bending (60/60; two trials). However, in embryos treated with $0.050 \mu \mathrm{M}$ SB431542 we observed randomized tail bending (37/70 left; 33/70 right; two trials). Intriguingly, this treatment did not impact juvenile heart asymmetry. In both DMSO controls and $0.050 \mu \mathrm{M}$ SB431542-treated samples, the heart consistently formed on the right side (50/50, DMSO; 70/70, SB431542; two trials).

To more strongly inhibit Nodal signaling, we treated Ciona neurula stage embryos with $5 \mu \mathrm{M}$ SB431542. In previous studies, this dosage disrupted left/right asymmetry in Ciona and other invertebrate embryos [25, $50,51]$ and we confirmed that this treatment abrogated Nodal-dependent lateral pitx expression (Additional file 1: Figure S1). In accordance with previous reports [50], we found that this treatment also led to loss of pigment spot asymmetry in the larval cerebral vesicle (data not shown). However, at this dosage embryos were unable to complete metamorphosis and juvenile heart positioning could not be assessed. We therefore examined the position of transgenically labeled heart progenitors in larval stages. To label the heart progenitors, zygotes were dechorionated and electroporated with Mesp > H2B:GFP [33]. Because dechorionation randomizes lateral heart directionality, we could not use this assay to characterize the role of Nodal signaling in Ciona lateral heart field directionality. However, we were still able to assess whether Nodal signaling was required for heart field asymmetry. Transgenic Mesp > H2B:GFP larvae were fixed at St. 29 and stained with phalloidin to visualize epidermal structures (Fig. 2a). We quantitatively assessed the degree to which heart progenitors were asymmetrically positioned by generating a displacement ratio for each sample (Fig. 2b). Based on ratios observed in wild-type larvae, larvae displaying heart displacement ratios of 1.2 or greater were classified as normal. Those with ratios of 1.1-1.2 were classified as having reduced asymmetric positioning, and those with ratios less than 1.1 were classified as having symmetric positioning. In accordance with our data from lower doses of SB431542, we found no discernible difference between $5 \mu \mathrm{M}$ SB431542-treated larva and DMSO controls (Fig. 2a", c). Together, these data suggest that Ciona lateral heart asymmetry is regulated through a chorion and Nodal-independent pathway.

\section{Neurula stage $\mathrm{H}^{+} / \mathrm{K}^{+}$-ATPase function is required} for asymmetric heart positioning and directionality The apparent Nodal independence of heart lateral asymmetry led us to search for alternative mechanisms. $\mathrm{H}^{+} /$ $\mathrm{K}^{+}$-ATPase-mediated ion flux contributes to left/right patterning in a variety of phyla, including the tunicates $[15-17,20]$. We therefore investigated whether $\mathrm{H}^{+} / \mathrm{K}^{+}$ATPase function contributes to Ciona heart lateral asymmetry. Chorionated embryos were treated with the $\mathrm{H}^{+}$/ $\mathrm{K}^{+}$-ATPase inhibitor omeprazole at the early neurula stage (St. 14) and then rinsed at late tailbud stages (St. 23). We first examined the impact of omeprazole treatment on tail bending. In contrast to DMSO controls (53/53, right, three trials), omeprazole treatment randomized tail bending (43/84, left; 41/84, right, three trials). This result aligns with previous data, indicating that ion flux regulates Ciona embryonic LR patterning [17].

We next examined the role of $\mathrm{H}^{+} / \mathrm{K}^{+}$-ATPase in lateral heart positioning. Early neurula stage embryos (St. 14) were treated with omeprazole and reared through metamorphosis. In omeprazole-treated juveniles, heart position was randomized (61/116 right, 38/116 left, three trials). Strikingly, we also observed that $15 \%$ of treated embryos developed into juveniles with medial hearts (17/116, Fig. $\left.2 d^{\prime}-d^{\prime \prime \prime \prime}\right)$. We also examined the impact of omeprazole treatment on larval heart precursor positioning. We found that omeprazole treatment had a dramatic, significant impact on heart precursor laterality with $>60 \%$ of treated larvae displaying symmetric positioning of the heart precursor field (Fig. 2a"', c). Taken together, these data suggest that ion flux regulates both lateral heart asymmetry and directionality.

Next, we investigated when ion flux is required for lateral heart asymmetry. Studies in Xenopus suggest that ion flux may contribute to left/right patterning as early as the fourcell stage [15]. In Ciona, a recent report indicates that neurula stage disruption of ion flux results in a dramatic and significant disruption of asymmetric pitx expression. These authors also showed that disruption of ion flux from fertilization until the end of gastrulation had a mild, but significant influence on asymmetric pitx expression [17]. To examine if there is an earlier role for ion flux in lateral heart asymmetry, we treated newly fertilized Ciona zygotes with omeprazole and rinsed off the inhibitor at the end of gastrulation (St. 13). This early omeprazole treatment had no impact on heart positioning (50/50, right, two trials). We also compared heart positioning in juveniles where omeprazole was applied exclusively during the neurula stages (St. 14-St. 16) with juveniles where omeprazole was applied during tailbud stages (St. 18-St. 23). As previously observed, neurula stage treatment with omeprazole randomized heart position and produced some juveniles with medial hearts ( $7 / 50$, two trials). In contrast, treatment with omeprazole during tailbud stages had no impact on heart positioning (50/50, right, two 


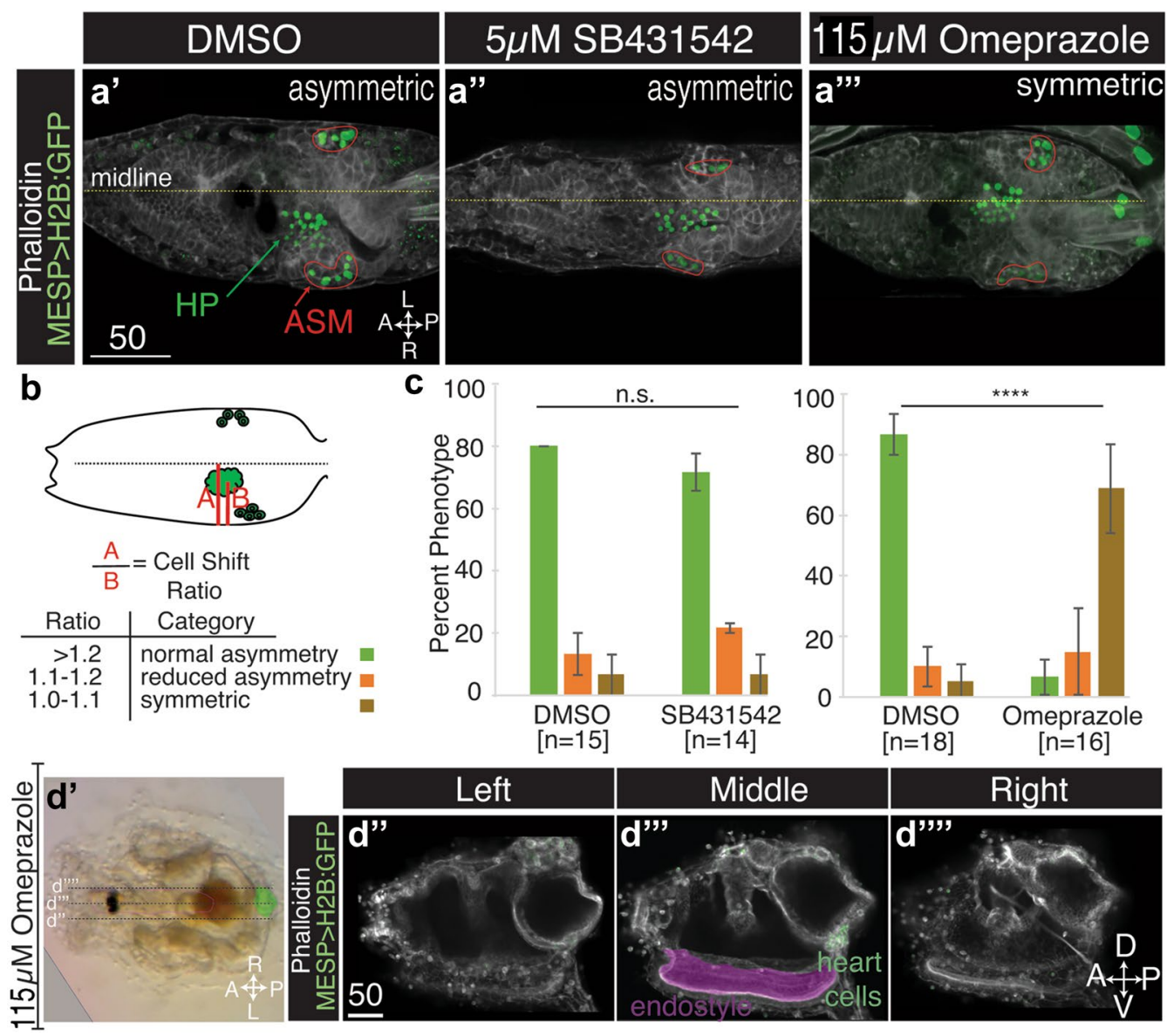

Fig. 2 Ion flux is required for laterally asymmetric heart positioning. $\mathbf{a}^{\prime}-\mathbf{a}^{\prime \prime \prime}$ Ventral projections of transgenic Stage 31 larva, and heart founder lineage cells are demarcated by Mesp > H2B:GFP (green), including heart precursors (HP) and atrial siphon muscle precursors (ASM). $\mathbf{a}^{\prime}-\mathbf{a}^{\prime \prime \prime}$ Typical asymmetric heart precursor positioning in DMSO controls $\left(\mathbf{a}^{\prime}\right)$, larva treated with $5 \mu \mathrm{M}$ SB431542 $\left(\mathbf{a}^{\prime \prime}\right)$ or larva treated with $115 \mu \mathrm{M}$ omeprazole $\left(\mathbf{a}^{\prime \prime \prime}\right)$. b Schematic illustrating cell shift ratio used for scoring heart precursor positioning, as detailed in the "Methods" section. c Graphical representation of larval heart position data. Error bars represent SEM, and $n$ represents total number of larva scored over three-independent trials. Chi-square test derived $P$ values comparing control and omeprazole-treated larva $(p=2.9 \mathrm{E}-5)$ or SB431542-treated samples $(p=0.84)$. $\mathbf{d}^{\prime}-\mathbf{d}^{\prime \prime \prime \prime \prime}$ Dorsal view $\left(\mathbf{d}^{\prime}\right)$ and lateral sections $\left(\mathbf{d}^{\prime \prime}-\mathbf{d}^{\prime \prime \prime \prime}\right)$ of Ciona juveniles displaying a midline heart phenotype following omeprazole treatment. In ( $\left.\mathbf{d}^{\prime}\right)$, the heart is falsecolored green and the endostyle is false-colored purple. In lateral sections $\left(\mathbf{d}^{\prime \prime}-\mathbf{d}^{\prime \prime \prime \prime}\right)$, heart cell nuclei are labeled by Mesp $>$ H2B:GFP. In all images, anterior is to the left. All scale bars are in micrometers

trials). These data suggest that ion flux specifically regulates heart asymmetry during neurula stages.

\section{Ion flux regulates neurula rotation}

We next examined whether ion flux regulates lateral heart asymmetry through an impact on neurula rotation. During late neurula stages, Ciona embryos undergo a rotation around the anterior-posterior axis (Fig. 1d). To determine whether the temporal overlap between sensitivity to omeprazole treatment (see previous section) and neurula stage rotation reflects a functional link, we treated embryos with omeprazole and assayed rotation. Embryos were treated with omeprazole or DMSO just prior to neurula rotation (St. 14) and subjected to timelapse video analysis. In control embryos, a clockwise rotation was observed. This rotation lasted until early tailbud stages and typically involved two-and-a-half turns along the axis (Additional file 2: video S1, left panel). In contrast, treated embryos show no sign of rotation (Additional file 2: video S1, right panel).

\section{Ion flux and chorion-dependent mechanisms synergistically regulate heart laterality}

To further examine the respective contributions of ion flux and chorion-dependent mechanisms, we treated dechorionated zygotes with omeprazole. While treatment of chorionated embryos produces midline hearts in $\sim 15 \%$ of juveniles, the combination of omeprazole treatment and dechorionation led to a dramatic increase in the penetrance of the midline heart phenotype $(\sim 52 \%)$. 
This increased penetrance aligns with our results on larval heart progenitor positioning in dechorionated embryos treated with omeprazole (Fig. 2c). Taken together, our results support a model in which ion flux has a dual impact on heart positioning. By influencing neurula rotation, ion flux helps to promote consistent asymmetric chorion contact, which then influences heart asymmetry. Additionally, ion flux appears to direct lateral heart asymmetry through an unknown, chorion-independent mechanism.

\section{Ion flux is required for polarized ciliogenesis}

We next began to investigate whether ion flux promotes Ciona neurula rotation by regulating polarized ciliogenesis. Previous studies have established that uniform cilia positioning in the vertebrate organizer is essential for generating flow [52]. Additionally, previous reports indicate that cilia are posteriorly positioned on Ciona epidermal cells during neurula stages [45]. Furthermore, recent work has demonstrated that ion flux is essential for ciliary growth and polarization in the Xenopus organizer [16]. To begin exploring whether ion flux plays a similar role in Ciona, we examined the morphology and localization of cilia during mid-neurula, late neurula and early tailbud stages using antibodies for acetylated alpha and gammatubulin (to label ciliary microtubules and centrioles, respectively, Fig. 3). Strikingly, the morphology and position of epidermal cilia appear to reflect well-characterized epidermal cell divisions during these stages (Fig. $3 \mathrm{a}^{\prime}-\mathrm{a}^{\prime \prime \prime \prime}$ ) [53]. At mid-neurula stage, epidermal cells are known to undergo a coordinated division [53]. As epithelial cells exit mitosis, an individual cilium forms on each daughter cell. These newly formed cilia are initially positioned as doublets spanning recently divided cell pairs (Fig. 3a', $\left.\mathrm{a}^{\prime \prime \prime \prime \prime}\right)$. A similar pattern has been observed during division in cultured mammalian cells [54]. These immature cilia are unlikely to generate a coherent flow. In late neurula stage embryos, epidermal cells do not divide. As recently formed cilia mature, they become positioned posteriorly along the cell's apical surface and display increased length in comparison with cilia in newly divided cells (Fig. 3a"). These mature, polarized cilia may generate a flow associated with the initial embryonic rotation at this stage. Lastly, at the early tailbud stage, epidermal cells initiate an uncoordinated wave of cell divisions [53]. Epidermal cilia are resorbed in patches where cells are entering mitosis and then reform as cells exit mitosis. Resorbed cilia as well as newly formed, immature cilia are unlikely to generate flow, and this could explain why tailbud embryos no longer rotate (Fig. 3a'"). Thus, we propose that cilia-dependent flow is temporally regulated by division-dependent ciliogenesis, prompting rotation at the late neurula stage and ending rotation at the early tailbud stage (Fig. $\left.3 \mathrm{a}^{\prime \prime \prime \prime}\right)$.
We next determined if ion flux is required for proper epidermal ciliogenesis in neurula stage embryos. We treated embryos with either DMSO or $115 \mu \mathrm{M}$ omeprazole beginning at the early neurula stage (St. 14). To assay for proper ciliogenesis, we fixed embryos at the late neurula stage (St. 16). At the onset of neurula rotation (St. 16), 75\% of epidermal cilia in control embryos displayed typical planar polarization (Fig. 3b", d). We also found that cilia had an average length of $1.6 \mu \mathrm{m}$ in control embryos (Fig. 3b", e). In embryos treated with omeprazole, cilia were often positioned close to the cell's center and only $45 \%$ of cilia displayed posterior polarization (Fig. 3c" $\mathrm{c}^{\prime \prime}$ ). Additionally, omeprazole treatment led to a significant decrease in cilia length (average length of $1.3 \mu \mathrm{m}$ in treated samples vs. $1.6 \mu \mathrm{m}$ in controls, Fig. $3 \mathrm{c}^{\prime \prime}$, e). These data support a model in which ion flux polarizes newly formed cilia in epidermal cells undergoing a coordinated mitotic wave during the mid-neurula stage. According to our model, these polarized cilia then generate a flow underlying neurula rotation.

\section{Lack of directional protrusion in shifting heart progenitors} The initial asymmetric positioning of the heart progenitors (St. 25) may result from active migration or may reflect a passive shift. To distinguish between these possibilities, we performed live imaging of heart progenitor membrane dynamics using a Mesp > GPI:GFP reporter [55]. We were able to record heart progenitor position and morphology from Stages 24-27, spanning their initial shift off of the midline. Although this shift was consistently observed between Stage 24 (Fig. 4a') and Stage 26 (Fig. $4 \mathrm{a}^{\prime \prime}$ ), we did not observe any protrusive activity during this period (Additional file 2: video S2, Additional files 3: video S3, Additional files 4: video S4). In Stage 27 larvae, protrusive activity was sometimes observed in lateral heart progenitor cells (Fig. 4a'"). However, this protrusive activity did not consistently correspond to the direction of the lateral shift (Additional file 2: video S2, Additional files 3: video S3, Additional files 4: video S4) and may be associated with migration of the atrial siphon muscle precursors [33]. Based on these observations, we propose that asymmetric heart positioning is a passive process, perhaps driven by adhesion to neighboring tissues that are undergoing directed lateral migration or torsion.

\section{Lateralized endoderm morphogenesis requires ion flux}

We searched for laterally asymmetric organs that could be driving the asymmetric shift observed in heart cells. Previous studies have detailed Ciona endoderm lateral asymmetries in larvae and juveniles, including rightsided positioning of the esophagus and stomach [56]. However, these studies have not characterized when 

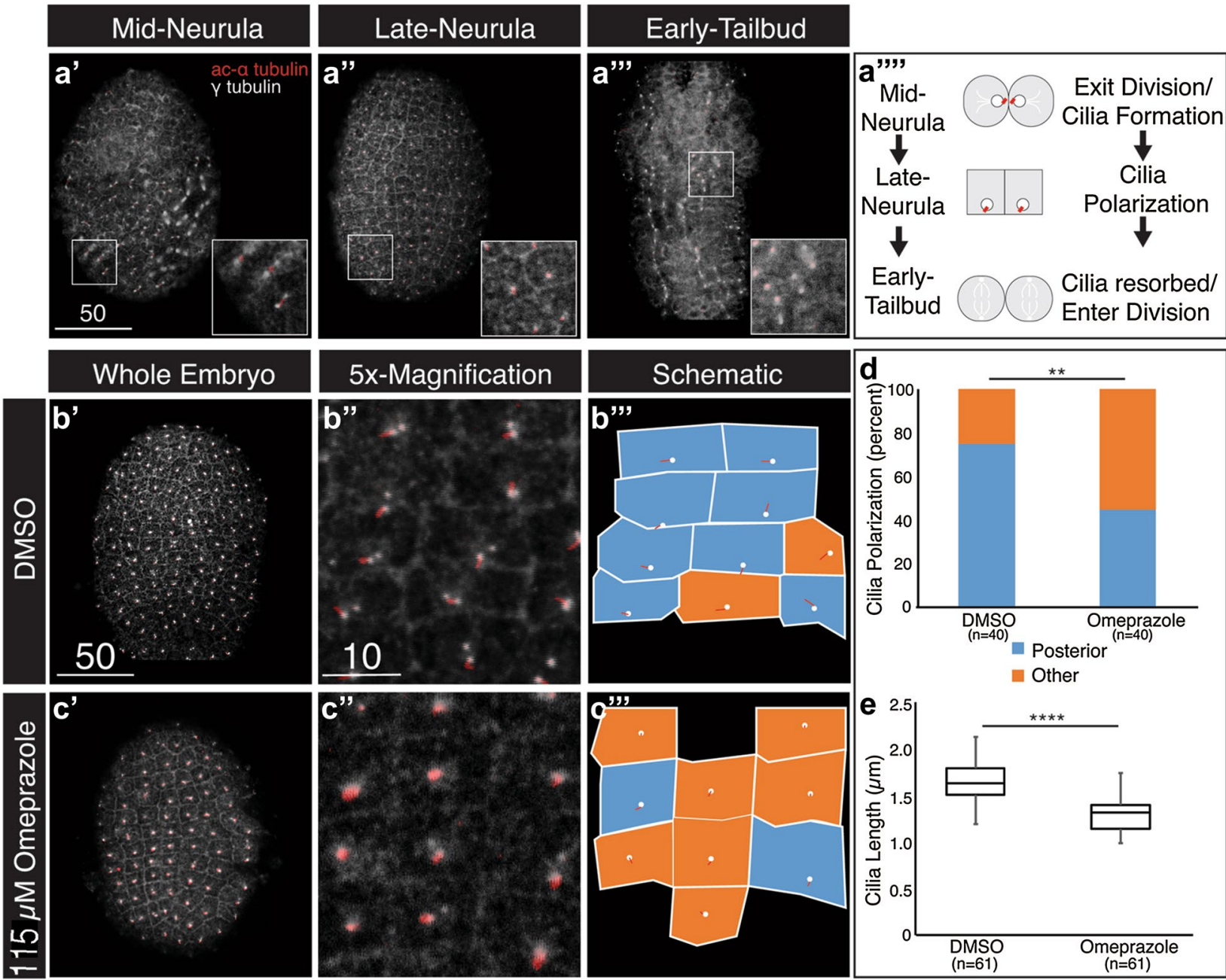

Fig. 3 Ion flux regulates ciliogenesis in the neurula epidermis. a-c Confocal images of neurula and tailbud stage embryos stained with antibodies against acetylated alpha-tubulin (red) and gamma-tubulin (white) to demarcate ciliary microtubules and centrioles, respectively. $\mathbf{a}^{\prime}-\mathbf{a}^{\prime \prime \prime}$ Insets show magnification of selected regions. $\mathbf{a}^{\prime \prime \prime \prime}$ Schematic showing cilia positioning during mid-neurula to early tailbud stages. $\mathbf{b}^{\prime}-\mathbf{c}^{\prime}$ Typical cilia positioning

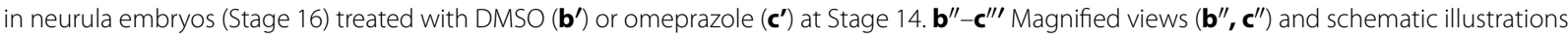
$\left(\mathbf{b}^{\prime \prime \prime}, \mathbf{c}^{\prime \prime \prime}\right)$ of cilia positioning. Blue coloration indicates posteriorly polarized cilia, and orange coloration indicates lack of posterior polarization (see "Methods" for details on scoring). d Graphical representation of data on cilia polarization in DMSO- and omeprazole-treated embryos. Colors correspond to $\left(\mathbf{b}^{\prime \prime \prime}, \mathbf{c}^{\prime \prime \prime}\right), n=$ number of cells scored from seven embryos over two independent trials. Chi-square derived $P$ value (0.006) for comparisons between DMSO versus Omeprazole-treated embryos. e Average cilia length in DMSO- and omeprazole-treated embryos. Box-and-whisker plot is shown. $n$ represents the number of cells scored in seven embryos over two independent trials; Wilcoxon rank sum test was used to derive $P$ value (3.3E-14) for comparisons between DMSO- versus omeprazole-treated embryos. In all images, anterior is up. Scale bars are in micrometers

endoderm asymmetry initially arises. To determine if Ciona heart asymmetry correlates temporally with endodermal asymmetry, we examined endoderm morphology in staged, phalloidin-stained samples. In these samples, endodermal rudiments are clearly delimited by columnar epithelia cells with apically enriched actin and trapezoidal hinge cells associated with folding (Fig. 5) [56]. In late tailbud II embryos (St. 24), the endoderm rudiment is roughly symmetric (Fig. $5 \mathrm{a}^{\prime}$ ). In ventral sections, the endoderm typically displays equal sized, bifurcated lateral out-pocketings. By St. 25, the ventral out-pocketing on the right bends toward the posterior while the outpocketing on the left bends toward the anterior (Fig. $5 \mathrm{a}^{\prime \prime}$ ). The right-sided out-pocketing is also distinguished at this stage by a more elongated, semicircular shape and appears to consist of taller, more trapezoidal shaped epithelial cells. Actin distribution on the right-sided epithelial cells was also more uniform and apically enriched. By St. 29, the right-sided ventral out-pocketing appears to elongate posteriorly (Fig. 5a'"). By contrast, the left-sided 


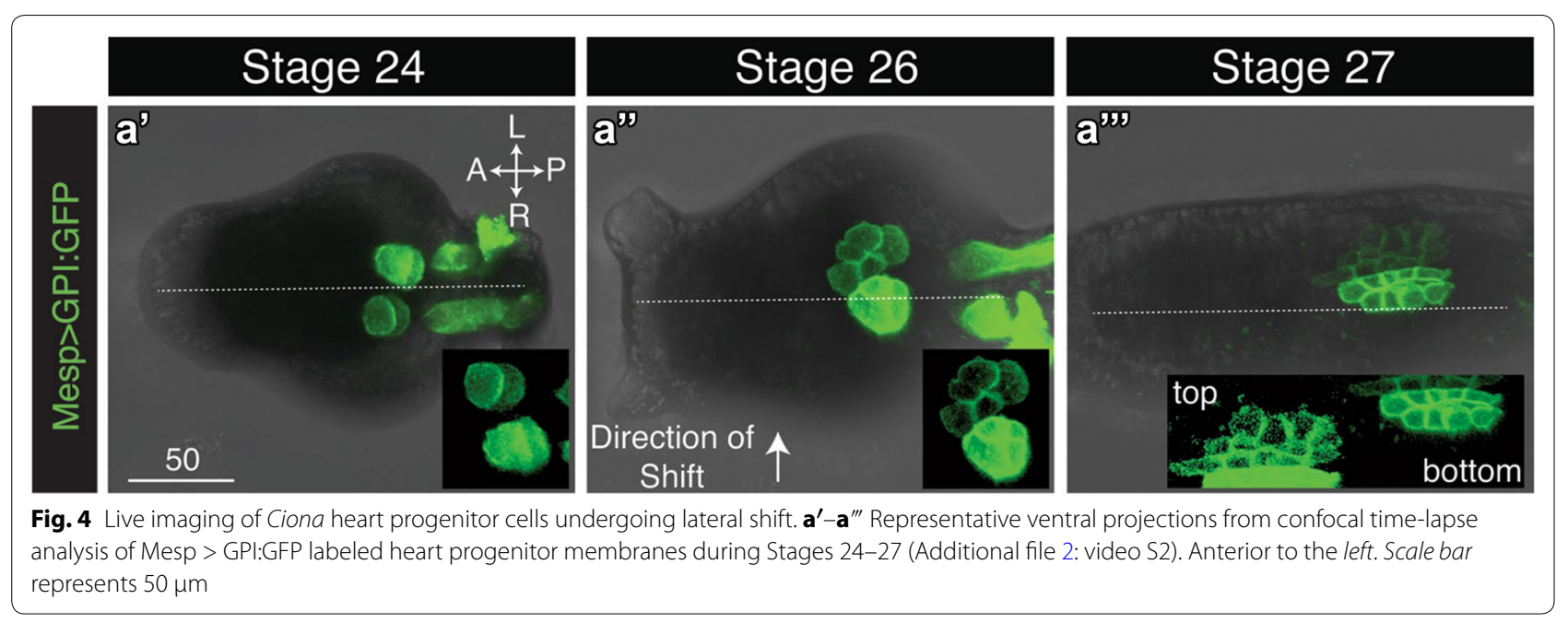

ventral out-pocketing appears to form an enclosed tubelike structure. These changes become more apparent as the larvae progress to St. 31 (Fig. 5b'). By this stage, rightand left-sided endodermal out-pocketings closely resemble previously characterized primordial stomach and intestine rudiments, respectively [56]. Thus, it appears that the initial lateral asymmetries in the heart and endoderm occur simultaneously at St. 25 , supporting a model in which asymmetric endoderm morphogenesis drives heart laterality.

If endoderm morphogenesis drives heart laterality, the observed ion flux dependence and Nodal independence of heart lateral asymmetry (Fig. 2) reflect properties of endoderm morphogenesis. To test this prediction, we disrupted Nodal signaling at the neurula stage $(5 \mu \mathrm{M}$ SB431542, St. 14.5) and assayed endodermal laterality in St. 31 larvae. Larvae treated with DMSO displayed typi$\mathrm{cal}$, asymmetric endodermal morphology (Fig. $5 \mathrm{~b}^{\prime}, \mathrm{c}$ ). In treated larvae, ventral endodermal rudiment morphology was severely disrupted and endoderm asymmetry was highly variable (Fig. 5b", c). Additionally, epithelial cells in SB431542-treated larvae appeared to be shorter, smaller and more densely packed with atypical, basolateral actin enrichment (Fig. 5b"). Although SB431542treated larvae sometimes displayed reduced asymmetry, it is difficult to determine whether this phenotype is due to disruption of left/right patterning or reflects general defects in endoderm morphogenesis.

We next examined the role of ion flux in lateral endoderm asymmetry. Neurula stage embryos (St. 14) treated with $115 \mu \mathrm{M}$ omeprazole were assayed for larval and juvenile endoderm morphology. In larvae treated with omeprazole, endodermal asymmetry was often reduced or absent. In particular, ventral out-pocketings were often similar on both left and right sides (Fig. $5 b^{\prime \prime \prime}$ ). This result was robust and significant $(\sim 45 \%$ symmetric, 9/20; Fig. 5c). Despite the observed loss of asymmetry in treated larvae, endodermal epithelial cells displayed normal shape and apical actin enrichment (Fig. $\left.5 b^{\prime \prime}\right)$. To quantify juvenile endoderm asymmetry, we scored stomach position and found consistent right-sided stomach positioning in all DMSO-treated juveniles (30/30, right, two trials; Fig. $\left.5 \mathrm{~d}^{\prime}-\mathrm{d}^{\prime \prime \prime}\right)$ [56]. In omeprazole-treated juveniles, we often observed randomized stomach positioning (25/72, left; 36/72, right, two trials). In some treated juveniles, we also observed formation of a presumed esophagus along the midline anterior to a large cavity, which we categorized as a midline stomach (Fig. $5 \mathrm{e}^{\prime}-\mathrm{e}^{\prime \prime \prime}$, 11/72, two trials). Additionally, in all juveniles scored, heart and stomach position was tightly correlated (data not shown), suggesting a causal link between lateral heart and endoderm asymmetry. Taken together, these data suggest that ion flux directly influences endoderm laterality, thereby indirectly promoting a lateral shift in the associated heart field.

\section{Discussion}

Based on our data, we have devised a model for directional asymmetry of the Ciona heart and endoderm (Fig. 6a). According to our model, ion flux regulates epidermal planar cell polarity (PCP) during neurulation. PCP serves to coordinate uniform cilia positioning required for flow-dependent neurula rotation and lateral chorion contact. Chorion contact triggers asymmetric Nodal signaling and Nodal-dependent embryonic asymmetric morphogenesis. Chorion contact may also contribute to lateral heart asymmetry in a Nodalindependent manner. We hypothesize that ion flux/PCP also contributes to lateral heart asymmetry independent of both chorion contact and Nodal signaling. Further, we 

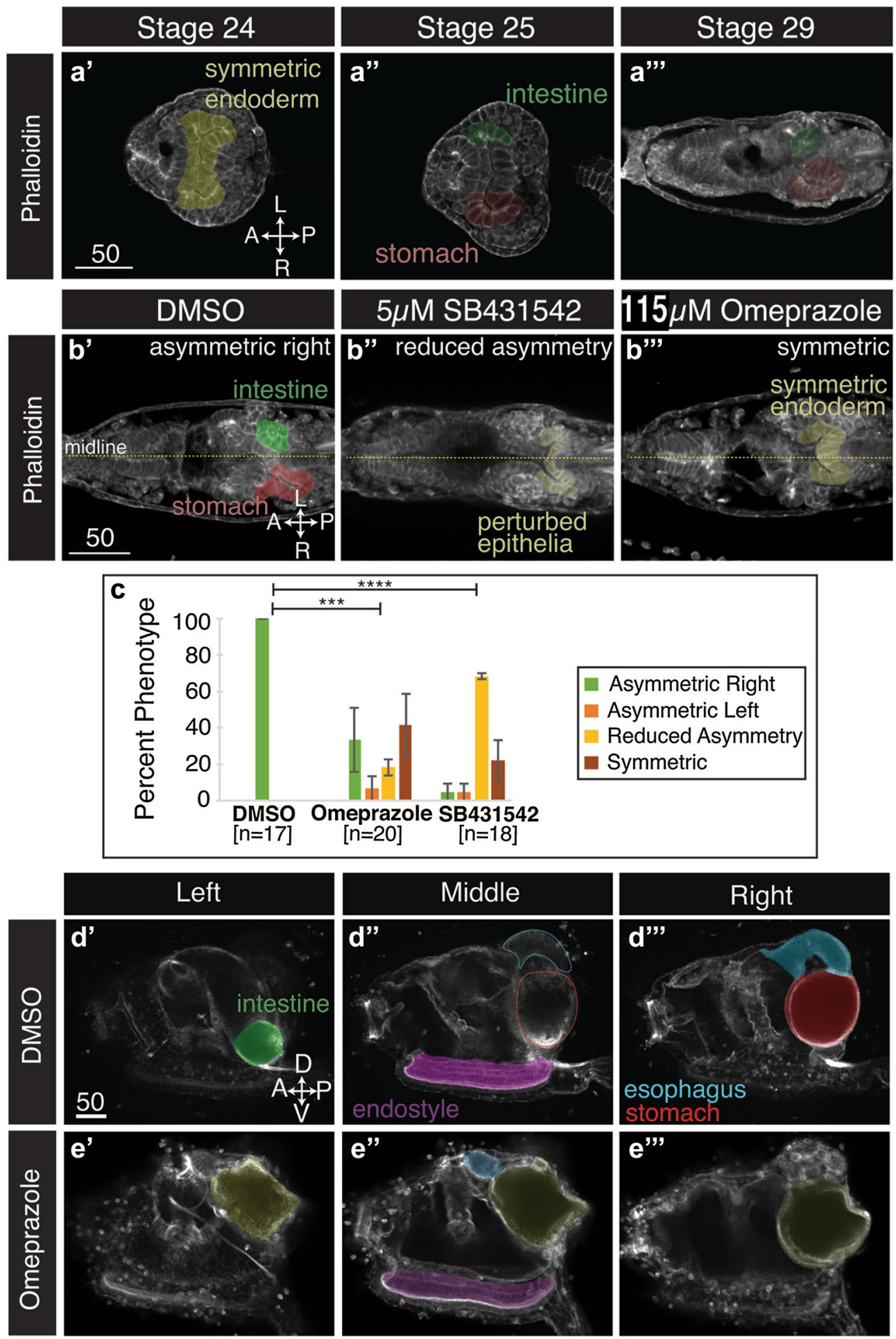

Fig. 5 Ion flux is required for proper endoderm asymmetry. $\mathbf{a}^{\prime}-\mathbf{a}^{\prime \prime \prime}$ Ventral sections of late embryonic and larval stages showing typical positioning of endodermal structures (false-colored). Cell outlines are visualized using phalloidin. $\mathbf{b}^{\prime}$ - $\mathbf{b}^{\prime \prime \prime}$ Ventral views of Stage 31 larvae, and endodermal structures are false-colored. $\mathbf{b}^{\prime}$ Typical endodermal morphology in DMSO controls. b" Typical endodermal morphology in larvae treated with the Nodal inhibitor SB431542. b"' Typical endodermal morphology in larvae treated with the ion flux inhibitor omeprazole. c Graphical representation of data on larval endodermal asymmetry. Error bars represent SEM. $n=$ number of larvae scored over three-independent trials and $x^{2}$ test derived $P$ values comparing control and omeprazole-treated larva $(p=6.9 \mathrm{E}-4)$ or SB431542-treated samples $(p=7.6 \mathrm{E}-7)$. $\mathbf{d}^{\prime}-\mathbf{e}^{\prime \prime \prime}$ Lateral sections of Ciona juveniles displaying endoderm asymmetry following DMSO $\left(\mathbf{d}^{\prime}-\mathbf{d}^{\prime \prime \prime}\right)$ and omeprazole neurula stage treatment $\left(\mathbf{e}^{\prime}-\mathbf{e}^{\prime \prime \prime}\right)$. Endodermal structures are false-colored. Yellow indicates symmetric endodermal organ. All scale bars are in micrometers 


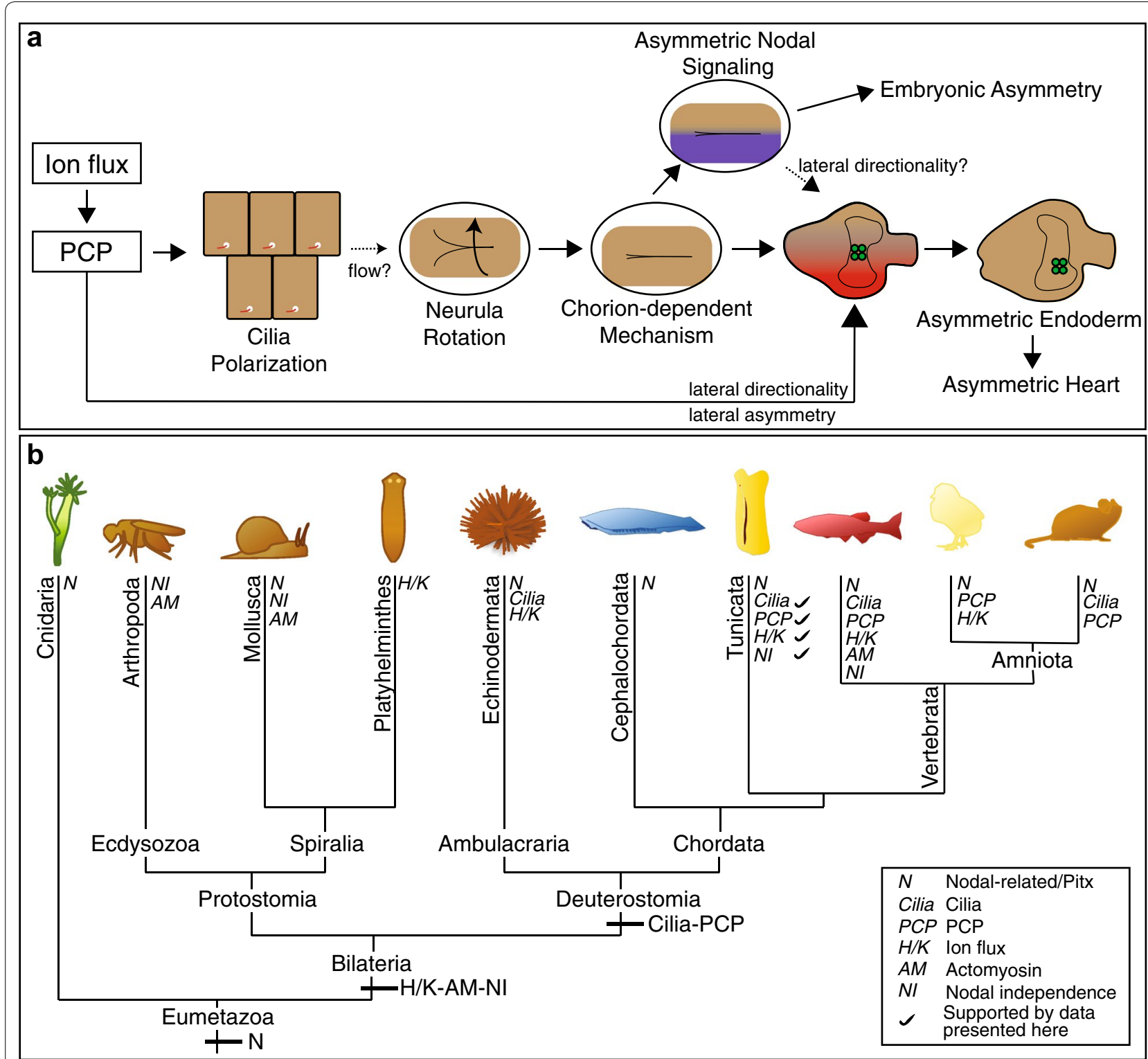

Fig. 6 Heart asymmetry in Ciona and other deuterostomes. a Model for lateral heart and endoderm asymmetry in Ciona. Solid arrows indicated support from experimental evidence while dotted arrows indicated more speculative hypotheses. b Model for eumetazoan evolution of left/right patterning

propose that lateralized morphogenesis of the endoderm rudiment underlies an associated lateral shift in heart progenitor position. We discuss each part of this model in the following sections.

\section{Ion flux-dependent PCP regulates cilia polarization}

Based on our results and the relevant literature, we hypothesize that ion flux influences PCP-dependent cilia polarization in the Ciona neurula epidermis through a direct impact on Wnt signaling. We have shown that blocking $\mathrm{H}^{+} / \mathrm{K}^{+}$ion flux disrupts cilia polarization (Fig. 3) and PCP is well established as the primary driver of cilia polarization [57-59]. The link between ion flux, PCP and cilia is best studied in relation to Xenopus LR patterning. In Xenopus embryos, ion flux is essential for proper cilia polarization in the organizer epithelium (gastrocoel roof plate) [16]. Since Wnt signaling requires an acidic extracellular environment $[60,61]$ and is known to regulate PCP [62], a possible link between ion flux, Wnt, PCP and cilia polarization was recently explored in Xenopus. It was found that knockdown of the $\mathrm{H}^{+} / \mathrm{K}^{+}$ion pump (ATP4) disrupted both canonical and non-canonical Wnt signaling and resulted in the loss of cilia polarization. In chick, the initial symmetry breaking is cilia independent, 
involving leftward cell movements in Hensen's node [14]. Directional cell movement requires ion flux and is dependent on proper PCP $[14,63]$. Thus, it appears that ion flux-dependent PCP may initiate lateral asymmetry in both frog and chick. An alternative, early role for ion flux in Xenopus involves shifts in membrane potential along the left/right axis during early cleavage stages [15]. However, in Ciona, we have shown that ion flux is not required for heart lateral asymmetry prior to neurulation.

\section{Contributions of epidermal cilia to lateral asymmetry}

We propose that polarized epidermal cilia have two distinct roles in LR patterning, serving to generate flow for neurula rotation and subsequently participating in chorion contact-mediated lateralization. Our data indicate that epidermal cilia mature and polarize precisely at the time when rotation occurs (Fig. 3), and previous studies indicate that epidermal cilia are motile at this stage [64]. Thus, epidermal cilia acquire the capability to generate flow-dependent rotation only during the neurula stage. Intriguingly, our data indicate that the timing of ciliadependent rotation is dictated by epidermal cell division patterns (Fig. 3). Following rotation, it is possible that cilia participate in chemosensory or mechanosensory functions associated with chorion contact. This dual role would mirror the established two-cilia model for left/ right patterning in vertebrate embryos. In mice, frogs and fish, motile organizer cilia generate a directional flow that triggers non-motile cilia in an asymmetric manner, resulting in the LR asymmetric signaling cascade [5-7, 65-69].

\section{Nodal-independent contributions to lateral asymmetry}

The relative contributions of ion flux, chorion contact and Nodal signaling to LR patterning remain difficult to untangle. We found that inhibition of ion flux disrupted both lateral directionality and asymmetry while dechorionation and pharmacological inhibition of Nodal had no impact on lateral asymmetry (Fig. 2). These results suggest that ion flux contributes to a LR asymmetry pathway that is independent of both Nodal and chorion contact. However, we also found that dechorionation enhances the impact of ion flux inhibition on lateral asymmetry, suggesting overlapping roles for ion flux and chorion contact. Nodal-independent lateral asymmetries in vertebrate embryos appear to involve polarized cytoskeletal dynamics. In zebrafish, Nodal-independent cardiac dextral looping is regulated by tissue intrinsic polarization of myosin [27]. In chick, the initial lateral asymmetries in the morphogenesis of Hensen's node are also Nodal independent and appear to involve planar cell polarization of cytoskeletal dynamics [63]. Thus, we are interested in investigating whether Nodal-independent lateral asymmetries in Ciona heart and gut morphogenesis also involve polarized cytoskeletal dynamics. We are also interested in investigating how the initial ion fluxdependent/Nodal-independent asymmetries generated in neurula stage embryos are translated into subsequent lateralization of heart and gut morphogenesis.

\section{Relationship between lateral asymmetries in endoderm and heart morphogenesis}

We propose that ion flux-dependent lateralization of endoderm morphogenesis underlies a passive, rightward shift in heart field position. According to this model, heart progenitors are tightly adherent to the adjacent endoderm. Thus, active rightward out-pocketing of the endoderm (Fig. 5) leads to passive rightward displacement of the heart progenitors. In support of this model, heart progenitors display no directed protrusive activity while they are initially shifting off of the midline (Fig. 4). Further, the initial asymmetries in heart and endoderm rudiments arise simultaneously at St. 25 in an ion fluxdependent manner (Figs. 2, 4). Additionally, in St. 21 late tailbud stage embryos, prior to their lateral shift at St. 25 larvae, heart progenitors appear to be embedded in the endoderm rudiment [31] and this close physical proximity is maintained in later stages. Thus, continued adhesion to endoderm epithelial cells or associated matrix in larval stages may facilitate heart progenitor displacement.

\section{Evolution of LR patterning}

Based on our data and the published literature, we have drawn out an evolutionary model regarding ancestral modes of LR patterning within the eumetazoans (Fig. 6b). A Nodal-related/Pitx module is generally considered to play an ancestral role in eumetazoan LR patterning. However, accumulating evidence suggests that a Nodalindependent pathway, involving ion flux or tissue intrinsic cytoskeletal polarity, may also play a deeply conserved role in lateral asymmetry among the bilaterians $[1,4]$. Inhibitor studies indicate that ion flux contributes to LR patterning in both vertebrates and non-chordate deuterostomes, including tunicates and echinoderms [17, 70]. Additionally, there is some evidence that ion flux contributes to LR patterning in planarians [71]. As discussed earlier, zebrafish heart formation and the initial asymmetric morphogenesis of the chick node appear to involve Nodal-independent cytoskeletal polarization [14, 27]. In protostome molluscs, polarized cytoskeletal elements function upstream of laterally asymmetric Nodal signaling $[72,73]$. In the ecdysozoans, Drosophila and $C$. elegans, Nodal genes are absent [74] and lateral asymmetries are solely based on cell intrinsic cytoskeletal polarity $[75,76]$. Widespread molecular similarities in ion flux or cytoskeletal pathways contributing to lateral 
asymmetry among bilaterians would strengthen the case for ancestral acquisition of this pathway.

We further propose that cilia-dependent LR patterning may have arisen within the ancestral deuterostome lineage. A central role for cilia in mouse, zebrafish and frog LR patterning is well established $[5,7,67]$. Accumulating data from this and other studies are beginning to clarify the role of cilia in tunicate LR patterning. Recently, sea urchins have also been found to deploy cilia for LR patterning [77, 78]. The involvement of cilia in LR patterning in a wide range of deuterostomes suggests that this represents an ancestral mechanism that was lost in some vertebrates, including chick and pig embryos. To further test this proposed evolutionary model, future studies should focus on testing whether cilia influence LR patterning in amphioxus and hemichordates. Additionally, further characterization of the upstream and downstream factors linking cilia to other LR patterning components and whether these components are broadly conserved within the deuterostomes will be critical in delineating how evolutionary shifts in LR patterning contributed to diversification in this clade.

\section{Additional files}

Additional file 1: Figure S1. Impact of SB431542 treatment on pitx expression. In situ results showing typical pitx expression in the heads of tailbud stage embryos (St. 22) dechorionated shortly before fixation and treated as indicated. (a') Wild-type expression pattern. (a") 5- $\mu \mathrm{M}$ treatment leads to loss of lateral pitx expression in the epidermis and internal germ layers, and note that Nodal-independent expression in the anterior neural boundary (Yoshida and Saiga, 2008) is not impacted. (a'"') 0.05- $\mu \mathrm{M}$ treatment appears to reduce pitx expression, particularly in the left epidermis, but does not eliminate Nodal-dependent lateral expression. The Ci-pitx probe was generated using $T 7$ polymerase on the Ci-pitx cDNA in Gene Collection library clone GC30b01 (Satou et al., 2002) according to standard protocols. To ensure that dechorionation did not interfere with left-right patterning, embryos were dechorionated just prior to fixation using the same protocol as employed for zygotic dechorionation. Standard in-situ hybridization protocols were also employed (Cooley et al., 2011).

Additional file 2: video S1. Ion flux is required for neurula rotation. Time-lapse of DMSO-treated (left panel) and 40- $\mu$ M omeprazole-treated (right panel) neurula stage embryos. Rotation is apparent on the left, while rotation is absent on the right. Movement at the end of the video in the right panel is associated with tail morphogenesis.

Additional file 3: video S2. Live imaging of Ciona heart progenitor cells. Ventral projections from one of three confocal time-lapse videos of three different embryos expressing Mesp>GPI::GFP to label heart progenitors. No consistent protrusive activity is observed. Protrusive activity after heart progenitors have shifted is associated with ASMPs.

Additional file 4: video S3. Live imaging of Ciona heart progenitor cells. Ventral projections from one of three confocal time-lapse videos of three different embryos expressing Mesp>GPI::GFP to label heart progenitors. No consistent protrusive activity is observed. Protrusive activity after heart progenitors have shifted is associated with ASMPs.

Additional file 5: video S4. Live imaging of Ciona heart progenitor cells. Ventral projections from one of three confocal time-lapse videos of three different embryos expressing Mesp>GPI::GFP to label heart progenitors. No consistent protrusive activity is observed. Protrusive activity after heart progenitors have shifted is associated with ASMPS.

\section{Abbreviations}

LR: left/right; FGF: fibroblast growth factor; HPF: hours post-fertilization; PCP: planar cell polarity.

\section{Authors' contributions}

KP performed all of the experiments, took a leading role in their design and wrote the article. BD assisted with experimental design and helped to revise the article. Both authors read and approved the final manuscript.

\section{Acknowledgements}

The authors wish to thank Dr. Jeroen Bakkers (Hubrecht Institute) for raising the question of Ciona heart asymmetry and his extensive guidance and feedback throughout the project, Christina Cota (Swarthmore College) for her extensive technical advice and support throughout the project, Rachel Merz (Swarthmore College) for her guidance on experiments associated with ciliogenesis and Gurrein Madan (Swarthmore College) for her assistance in generating the pitx probe. We also thank Prof. Lynne Schofield (Swarthmore College) for assistance with statistical evaluation of our results.

\section{Competing interests}

The authors declare that they have no competing interests.

Availability of data and supporting materials

All plasmids and raw data are available upon request following publication.

\section{Funding}

Funding was provided by Swarthmore College and the NIH (1R01HL091027, R15 HD080525-01).

\section{Publisher's Note}

Springer Nature remains neutral with regard to jurisdictional claims in published maps and institutional affiliations.

Received: 31 May 2017 Accepted: 17 July 2017

Published online: 25 July 2017

\section{References}

1. Namigai EKO, Kenny NJ, Shimeld SM. Right across the tree of life: the evolution of left-right asymmetry in the Bilateria. Genesis. 2014;52:458-70. doi:10.1002/dvg.22748.

2. Peeters H, Devriendt K. Human laterality disorders. Eur J Med Genet. 2006;49:349-62. doi:10.1016/j.ejmg.2005.12.003.

3. Gilboa SM, Salemi JL, Nembhard WN, Fixler DE, Correa A. Mortality resulting from congenital heart disease among children and adults in the United States, 1999 to 2006. Circulation. 2010;122:2254-63. doi:10.1161/ CIRCULATIONAHA.110.947002.

4. Vandenberg LN, Levin M. A unified model for left-right asymmetry? Comparison and synthesis of molecular models of embryonic laterality. Dev Biol. 2013;379:1-15. doi:10.1016/j.ydbio.2013.03.021.

5. Nonaka S, Tanaka Y, Okada Y, Takeda S, Harada A, Kanai Y, Kido M, Hirokawa N. Randomization of left-right asymmetry due to loss of nodal cilia generating leftward flow of extraembryonic fluid in mice lacking KIF3B motor protein. Cell. 1998;95:829-37. doi:10.1016/ S0092-8674(00)81705-5.

6. Kramer-Zucker, AG, Olale, F, Haycraft, CJ, Yoder BK, Schier AF, Drummond IA. Cilia-driven fluid flow in the zebrafish pronephros, brain and Kupffer's vesicle is required for normal organogenesis. Development. 2005. http:// dev.biologists.org/content/132/8/1907.short. Accessed 16 Apr 2017.

7. Schweickert A, Weber T, Beyer T, Vick P, Bogusch S, Feistel K, Blum M. Ciliadriven leftward flow determines laterality in Xenopus. 2007. doi:10.1016/j. cub.2006.10.067.

8. Chen J, Knowles HJ, Hebert JL, Hackett BP. Mutation of the mouse hepatocyte nuclear factor/forkhead homologue 4 gene results in an absence of cilia and random left-right asymmetry. J Clin Invest. 1998;102:1077-82. http://www.jci.org. Accessed 23 Apr 2017.

9. Tanaka Y, Okada Y, Hirokawa N, Carson JL, Vrablic K, Gesteland K, Schoenwolf GC, Hirokawa N. FGF-induced vesicular release of Sonic hedgehog 
and retinoic acid in leftward nodal flow is critical for left-right determination. Nature. 2005:435:172-7. doi:10.1038/nature03494.

10. Fischer A, Viebahn C, Blum M. FGF8 acts as a right determinant during establishment of the left-right axis in the rabbit. Curr Biol. 2002;12:180716. doi:10.1016/S0960-9822(02)01222-8.

11. Meyers EN, Martin GR. Differences in left-right axis pathways in mouse and chick: functions of FGF8 and SHH. Science (80-.). 1999;285:403-6.

12. Brennan J, Norris DP, Robertson EJ. Nodal activity in the node governs left-right asymmetry. Genes Dev. 2002;16:2339-44. doi:10.1101/ gad. 1016202

13. McGrath J, Somlo S, Makova S, Tian X, Brueckner M. Two populations of node monocilia initiate left-right asymmetry in the mouse. Cell. 2003;114:61-73. doi:10.1016/50092-8674(03)00511-7.

14. Gros J, Feistel K, Viebahn C, Blum M, Tabin CJ. Cell movements at Hensen's node establish left/right asymmetric gene expression in the chick. Science (80-.). 2009. http://science.sciencemag.org/content/324/5929/941. Accessed 14 Apr 2017.

15. Levin M, Thorlin T, Robinson KR, Nogi T, Mercola M. Asymmetries in $\mathrm{H}^{+} / \mathrm{K}^{+}$-ATPase and cell membrane potentials comprise a very early step in left-right patterning. Cell. 2002;111:77-89. doi:10.1016/ S0092-8674(02)00939-X.

16. Walentek P, Beyer T, Thumberger T, Schweickert A, Blum M. ATP4a is required for Wnt-dependent Foxj1 expression and leftward flow in Xenopus left-right development. Cell Rep. 2012;1:516-27. doi:10.1016/j. celrep.2012.03.005.

17. Shimeld SM, Levin M. Evidence for the regulation of left-right asymmetry in Ciona intestinalis by ion flux. Dev Dyn. 2006;235:1543-53. doi:10.1002/ dvdy.20792.

18. Su YH. Telling left from right: left-right asymmetric controls in sea urchins. Genesis. 2014;52:269-78. doi:10.1002/dvg.22739.

19. Levin M, Johnson RL, Sterna CD, Kuehn M, Tabin C. A molecular pathway determining left-right asymmetry in chick embryogenesis. Cell. 1995:82:803-14. doi:10.1016/0092-8674(95)90477-8.

20. Raya A, Belmonte JCl. Unveiling the establishment of left-right asymmetry in the chick embryo. Mech Dev. 2004;121:1043-54. doi:10.1016/j. mod.2004.05.005.

21. Blum M, Beyer T, Weber T, Vick P, Andre P, Bitzer E, Schweickert A. Xenopus, an ideal model system to study vertebrate left-right asymmetry. Dev Dyn. 2009;238:1215-25. doi:10.1002/dvdy.21855.

22. Long S, Ahmad N, Rebagliati M. The zebrafish nodal-related gene southpaw is required for visceral and diencephalic left-right asymmetry. Development. 2003;130:2303-16. doi:10.1242/dev.00436.

23. Soukup V, Yong LW, Lu T-M, Huang S-W, Kozmik Z, Yu J-K. The Nodal signaling pathway controls left-right asymmetric development in amphioxus. Evodevo. 2015;6:5. doi:10.1186/2041-9139-6-5.

24. Yoshida K, Saiga H. Left-right asymmetric expression of Pitx is regulated by the asymmetric Nodal signaling through an intronic enhancer in Ciona intestinalis. Dev Genes Evol. 2008;218:353-60. doi:10.1007/ s00427-008-0230-3.

25. Grande C, Patel NH. Nodal signalling is involved in left-right asymmetry in snails. Nature. 2009. doi:10.1038/nature07603.

26. Watanabe H, Schmidt HA, Kuhn A, Höger SK, Kocagöz Y, Laumann-Lipp N Zbek S, Holstein TW. Nodal signalling determines biradial asymmetry in Hydra. Nature. 2014. doi:10.1038/nature13666.

27. Noel ES, Verhoeven M, Lagendijk AK, Tessadori F, Smith K, Choorapoikayil S, den Hertog J, Bakkers J. A Nodal-independent and tissue-intrinsic mechanism controls heart-looping chirality. Nat Commun. 2013;4:1-9. doi:10.1038/ncomms3754.

28. Chen JN, van Eeden FJ, Warren KS, Chin A, Nusslein-Volhard C, Haffter P, Fishman MC. Left-right pattern of cardiac BMP4 may drive asymmetry of the heart in zebrafish. Development. 1997. http://dev.biologists.org/ content/124/21/4373.short. Accessed 23 Apr 2017.

29. Smith KA, Chocron S, von der Hardt S, de Pater E, Soufan A, Bussmann J, Schulte-Merker S, Hammerschmidt M, Bakkers J, Rotation and Asymmetric Development of the Zebrafish Heart Requires Directed Migration of Cardiac ProSmith KA, Chocron S, von der Hardt S, de Pater E, Soufan A, Bussmann J et al. Rotation and asymmetric development of the zeb. Dev Cell 2008;14:287-97. doi:10.1016/j.devcel.2007.11.015.

30. Lenhart KF, Holtzman NG, Williams JR, Burdine RD. Integration of Nodal and BMP signals in the heart requires Fox $\mathrm{H} 1$ to create left-right differences in cell migration rates that direct cardiac asymmetry. PLoS Genet. 2013. doi:10.1371/journal.pgen.1003109.

31. Davidson B. Ciona intestinalis as a model for cardiac development. Semin Cell Dev Biol. 2007;18:16-26. doi:10.1016/j.semcdb.2006.12.007.

32. Diogo R, Kelly RG, Christiaen L, Levine M, Ziermann JM, Molnar JL, Noden $D M$, Tzahor E. A new heart for a new head in vertebrate cardiopharyngeal evolution. Nature. 2015;520:466-73. doi:10.1038/nature14435.

33. Stolfi A, Gainous TB, Young JJ, Mori A, Levine M, Christiaen L. Early chordate origins of the vertebrate second heart field. Science. 2010;329:5658. doi:10.1126/science.1190181.

34. Lemaire P. Evolutionary crossroads in developmental biology: the tunicates. Development. 2011. http://dev.biologists.org/content/138/11/2143.short. Accessed 23 Apr 2017.

35. Lemaire P, Smith WC, Nishida H. Ascidians and the plasticity of the chordate developmental program. Curr Biol. 2008;18:R620-31. doi:10.1016/j. cub.2008.05.039.

36. Jiang D, Smith WC. Ascidian notochord morphogenesis. Dev Dyn. 2007:236:1748-57. doi:10.1002/dvdy.21184.

37. Satoh $N$. The ascidian tadpole larva: comparative molecular development and genomics. Nat Rev Genet. 2003:4:285-95. doi:10.1038/nrg1042.

38. Davidson B, Levine M. Evolutionary origins of the vertebrate heart: specification of the cardiac lineage in Ciona intestinalis. Proc Natl Acad Sci USA. 2003;100:11469-73. doi:10.1073/pnas.1634991100.

39. Satou Y, Imai KS, Satoh N. The ascidian Mesp gene specifies heart precursor cells. Development. 2004. http://dev.biologists.org/content/131/11/2533. Accessed 23 Apr 2017.

40. Christiaen L, Davidson B, Kawashima T, Powell W, Nolla H, Vranizan K, Levine M. The transcription/migration interface in heart precursors of Ciona intestinalis. Science (80-.). 2008. http://science.sciencemag.org/ content/320/5881/1349. Accessed 23 Apr 2017.

41. Davidson B, Shi W, Beh J, Christiaen L, Levine M. FGF signaling delineates the cardiac progenitor field in the simple chordate, Ciona intestinalis. Genes Dev. 2006:20:2728-38. doi:10.1101/gad.1467706.

42. Razy-Krajka F, Lam K, Wang W, Stolfi A, Joly M, Bonneau R, Christiaen L. Collier/OLF/EBF-dependent transcriptional dynamics control pharyngeal muscle specification from primed cardiopharyngeal progenitors. Dev Cell. 2014;29:263-76. doi:10.1016/j.devcel.2014.04.001.

43. Chea HK, Wright CV, Swalla BJ. Nodal signaling and the evolution of deuterostome gastrulation. Dev Dyn. 2005;234:269-78. doi:10.1002/ dvdy.20549.

44. Nishide K, Mugitani M, Kumano G, Nishida H. Neurula rotation determines left-right asymmetry in ascidian tadpole larvae. Development. 2012;139:1467-75. doi:10.1242/dev.076083.

45. Thompson H, Shaw MK, Dawe HR, Shimeld SM. The formation and positioning of cilia in Ciona intestinalis embryos in relation to the generation and evolution of chordate left-right asymmetry. Dev Biol. 2012;364:21423. doi:10.1016/j.ydbio.2012.02.002.

46. Brunetti R, Gissi C, Pennati R, Caicci F, Gasparini F, Manni L. Morphological evidence that the molecularly determined Ciona intestinalis type $\mathrm{A}$ and type B are different species: Ciona robusta and Ciona intestinalis. J Zool Syst Evol Res. 2015;53:186-93. doi:10.1111/jzs.12101.

47. Corbo JC, Levine M, Zeller RW. Characterization of a notochord-specific enhancer from the Brachyury promoter region of the ascidian, Ciona intestinalis. Development. 1997;124:589-602.

48. Hotta K, Mitsuhara K, Takahashi H, Inaba K, Oka K, Gojobori T, Ikeo K. A web-based interactive developmental table for the ascidian Ciona intestinalis, including 3D real-image embryo reconstructions: I. From fertilized egg to hatching larva. Dev Dyn. 2007;236:1790-805. doi:10.1002/ dvdy.21188.

49. Mita K, Fujiwara S. Nodal regulates neural tube formation in the Ciona intestinalis embryo. Dev Genes Evol. 2007;217:593-601. doi:10.1007/ s00427-007-0168-x.

50. Yoshida K, Saiga H. Repression of Rx gene on the left side of the sensory vesicle by Nodal signaling is crucial for right-sided formation of the ocellus photoreceptor in the development of Ciona intestinalis. Dev Biol. 2011:354:144-50. doi:10.1016/j.ydbio.2011.03.006.

51. Luo Y-J, Su Y-H, Bolouri H, He D, Davidson E. Opposing Nodal and BMP signals regulate left-right asymmetry in the sea urchin larva. PLoS Biol. 2012;10:e1001402. doi:10.1371/journal.pbio.1001402.

52. Marshall WF, Kintner C. Cilia orientation and the fluid mechanics of development. Curr Opin Cell Biol. 2008;20:48-52. doi:10.1016/j.ceb.2007.11.009. 
53. Ogura Y, Sasakura Y. Developmental control of cell-cycle compensation provides a switch for patterned mitosis at the onset of chordate neurulation. Dev Cell. 2016;37:148-61. doi:10.1016/j.devcel.2016.03.013.

54. Zhang B, Zhang T, Wang G, Wang G, Chi W, Jiang Q, Zhang C. GSK3ßDzip1-Rab8 cascade regulates ciliogenesis after mitosis. PLoS Biol. 2015;13:e1002129. doi:10.1371/journal.pbio.1002129.

55. Cooley J, Whitaker S, Sweeney S, Fraser S, Davidson B. Cytoskeletal polarity mediates localized induction of the heart progenitor lineage. doi:10.1038/ncb2291.

56. Nakazawa K, Yamazawa T, Moriyama Y, Ogura Y, Kawai N, Sasakura Y, Saiga $\mathrm{H}$. Formation of the digestive tract in Ciona intestinalis includes two distinct morphogenic processes between its anterior and posterior parts. Dev Dyn. 2013;242:1172-83. doi:10.1002/dvdy.24009.

57. Song H, Hu J, Chen W, Elliott G, Andre P, Gao B, Yang Y. Planar cell polarity breaks bilateral symmetry by controlling ciliary positioning. Nature. 2010. doi:10.1038/nature09129.

58. Maisonneuve C, Guilleret I, Vick P, Weber T, Andre P, Beyer T, Blum M, Constam DB. Bicaudal C, a novel regulator of Dvl signaling abutting RNA-processing bodies, controls cilia orientation and leftward flow. Development. 2009. http://dev.biologists.org/content/136/17/3019.long. Accessed 14 Apr 2017

59. Borovina A, Superina S, Voskas D, Ciruna B. Vangl2 directs the posterior tilting and asymmetric localization of motile primary cilia. Nat Cell Biol. 2010;12:407-12. doi:10.1038/ncb2042.

60. Cruciat C-M, Ohkawara B, Acebron SP, Karaulanov E, Reinhard C, Ingelfinger D, Boutros M, Niehrs C. Requirement of prorenin receptor and vacuolar $\mathrm{H}^{+}$-ATPase-mediated acidification for Wnt signaling. Science (80-.). 2010. http://science.sciencemag.org/content/327/5964/459. Accessed 14 Apr 2017.

61. Hermle T, Petzoldt AG, Simons M. The role of proton transporters in epithelial Wnt signaling pathways. Pediatr Nephrol. 2011;26:1523-7. doi:10.1007/s00467-011-1823-z.

62. Sokol SY. Spatial and temporal aspects of Wnt signaling and planar cell polarity during vertebrate embryonic development. Semin Cell Dev Biol. 2015;42:78-85. doi:10.1016/j.semcdb.2015.05.002.

63. Zhang Y, Levin M. Left-right asymmetry in the chick embryo requires core planar cell polarity protein Vangl2. Genesis. 2009;47:719-28. doi:10.1002/ dvg.20551.

64. Katsumoto S, Hatta K, Nakagawa M. Brief hypo-osmotic shock causes test cell death, prevents neurula rotation, and disrupts left-right asymmetry in Ciona intestinalis. Zool Sci. 2013;30:352-9. doi:10.2108/zsj.30.352.

65. Antic D, Stubbs JL, Suyama K, Kintner C, Scott MP, Axelrod JD. Planar cell polarity enables posterior localization of nodal cilia and left-right axis determination during mouse and Xenopus embryogenesis. PLoS ONE. 2010;5:e8999. doi:10.1371/journal.pone.0008999.

66. Nonaka S, Shiratori H, Saijoh Y, Hamada H. Determination of left-right patterning of the mouse embryo by artificial nodal flow. Nature. 2002;418:96-9. doi:10.1038/nature00849.

67. Essner JJ, Amack JD, Nyholm MK, Harris EB, Yost HJ. Kupffer's vesicle is a ciliated organ of asymmetry in the zebrafish embryo that initiates left-right development of the brain, heart and gut. Development. 2005 http://dev.biologists.org/content/132/6/1247.short. Accessed $16 \mathrm{Apr}$ 2017.

68. Tabin CJ, Vogan KJ. A two-cilia model for vertebrate left-right axis specification. Genes Dev. 2003;17:1-6. doi:10.1101/gad.1053803.

69. Hirokawa N, Tanaka Y, Okada Y. Cilia, KIF3 molecular motor and nodal flow. Curr Opin Cell Biol. 2012;24:31-9. doi:10.1016/j.ceb.2012.01.002.

70. Hibino T, Ishii Y, Levin M, Nishino A. Ion flow regulates left-right asymmetry in sea urchin development. Dev Genes Evol. 2006;216:265-76. doi:10.1007/s00427-005-0051-6.

71. Nogi T, Yuan Y, Sorocco D, Perez-Tomas R, Levin M, Nogi T, Emily Yuan Y, Sorocco D, Perez-Tomas R, Levin M. Eye regeneration assay reveals an invariant functional left-right asymmetry in the early bilaterian, Dugesia japonica. Laterality Aymmetries Body Brain Cogn. 2005;10:193-205. doi:10.1080/1357650054200001440.

72. Abe M, Takahashi H, Kuroda R. Spiral cleavages determine the left-right body plan by regulating Nodal pathway in monomorphic gastropods, Physa acuta. Int J Dev Biol. 2014;58:513-20. doi:10.1387/ijdb.140087rk

73. Davison A, McDowell GS, Holden JM, Johnson HF, Koutsovoulos GD, Liu MM, Hulpiau P, Van Roy F, Wade CM, Banerjee R, Yang F, Chiba S, Davey JW, Jackson DJ, Levin M, Blaxter ML. Formin is associated with left-right asymmetry in the pond snail and the frog. Curr Biol. 2016. doi:10.1016/j. cub.2015.12.071.

74. Grande C, Martin-Duran JM, Kenny NJ, Truchado-Garcia M, Hejnol A. Evolution, divergence and loss of the Nodal signaling pathway: new data and a synthesis across the Bilateria. Int J Dev Biol. 2014;58:521-32. doi:10.1387/ijdb.140133cg.

75. Taniguchi K, Maeda R, Ando T, Okumura T, Nakazawa N, Hatori R, Nakamura M, Hozumi S, Fujiwara H, Matsuno K. Chirality in planar cell shape contributes to left-right asymmetric epithelial morphogenesis. Science (80-.). 2011. http://science.sciencemag.org/content/333/6040/339.full. Accessed 23 Apr 2017.

76. Pohl C, Bao Z. Chiral forces organize left-right patterning in C. elegans by uncoupling midline and anteroposterior axis. Dev Cell. 2010;19:402-12. doi:10.1016/j.devcel.2010.08.014.

77. Takemoto A, Miyamoto T, Simono F, Kurogi N, Shirae-Kurabayashi M, Awazu A, Suzuki K-IT, Yamamoto T, Sakamoto N. Cilia play a role in breaking left-right symmetry of the sea urchin embryo. Genes Cells. 2016. doi:10.1111/gtc.12362.

78. Tisler M, Wetzel F, Mantino S, Kremnyov S, Thumberger T, Schweickert A, Blum M, Vick P. Cilia are required for asymmetric nodal induction in the sea urchin embryo. BMC Dev Biol. 2016;16:28. doi:10.1186/ s12861-016-0128-7.

\section{Submit your next manuscript to BioMed Central and we will help you at every step:}

- We accept pre-submission inquiries

- Our selector tool helps you to find the most relevant journal

- We provide round the clock customer support

- Convenient online submission

- Thorough peer review

- Inclusion in PubMed and all major indexing services

- Maximum visibility for your research

Submit your manuscript at www.biomedcentral.com/submit 